\title{
Research Article \\ Schur-Type Inequalities for Complex Polynomials with no Zeros in the Unit Disk
}

\author{
Szilárd Gy. Révész \\ Received 20 March 2007; Accepted 28 June 2007 \\ Recommended by Saburou Saitoh
}

Starting out from a question posed by T. Erdélyi and J. Szabados, we consider Schurtype inequalities for the classes of complex algebraic polynomials having no zeros within the unit disk $D$. The class of polynomials with no zeros in $D$-also known as Bernstein or Lorentz class_-was studied in detail earlier. For real polynomials utilizing the Bernstein-Lorentz representation as convex combinations of fundamental polynomials $(1-x)^{k}(1+x)^{n-k}$, G. Lorentz, T. Erdélyi, and J. Szabados proved a number of improved versions of Schur- (and also Bernstein- and Markov-) type inequalities. Here we investigate the similar questions for complex polynomials. For complex polynomials, the above convex representation is not available. Even worse, the set of complex polynomials, having no zeros in the unit disk, does not form a convex set. Therefore, a possible proof must go along different lines. In fact, such a direct argument was asked for by Erdélyi and Szabados already for the real case. The sharp forms of the Bernstein- and Markov-type inequalities are known, and the right factors are worse for complex coefficients than for real ones. However, here it turns out that Schur-type inequalities hold unchanged even for complex polynomials and for all monotonic, continuous weight functions. As a consequence, it becomes possible to deduce the corresponding Markov inequality from the known Bernstein inequality and the new Schur-type inequality with logarithmic weight.

Copyright (c) 2007 Szilárd Gy. Révész. This is an open access article distributed under the Creative Commons Attribution License, which permits unrestricted use, distribution, and reproduction in any medium, provided the original work is properly cited.

\section{Introduction}

Let $\mathscr{P}_{n}$ and $\mathscr{P}_{n}^{c}$ denote the set of one-variable algebraic polynomials of degree at most $n$ with real, respectively, complex coefficients, and denote the set of all the (real or complex) polynomials by $\mathscr{P}$ and $\mathscr{P} c$, respectively. The open unit interval will be denoted by 
2 Journal of Inequalities and Applications

$I:=(-1,1)$, and the open unit disk $\{z:|z|<1\}$ will be denoted by $D$. We take

$$
\|f\|:=\sup _{I}|f|
$$

as the norm of a polynomial or a continuous function.

In approximation theory, Schur- and Bernstein-type polynomial inequalities constitute an important subject, see, for example, $[1,2]$. The classical inequality of Schur states that

$$
\|p\| \leq(n+1)\left\|p(x) \sqrt{1-x^{2}}\right\| \quad\left(p \in \mathscr{P}_{n}\right)
$$

This can be generalized to weights $\left(1-x^{2}\right)^{\alpha}$ with $\alpha>0$ as well:

$$
\|p\| \leq C(\alpha) n^{2 \alpha}\left\|p(x)\left(1-x^{2}\right)^{\alpha}\right\| \quad\left(p \in \mathscr{P}_{n}\right) .
$$

Schur's inequality (1.2) is usually combined with Bernstein's inequality

$$
\left|p^{\prime}(x)\right| \leq \frac{n}{\sqrt{1-x^{2}}}\|p\| \quad\left(p \in \mathscr{P}_{n}\right)
$$

to deduce Markov's inequality

$$
\left\|p^{\prime}\right\| \leq n^{2}\|p\| \quad\left(p \in \mathscr{P}_{n}\right) .
$$

Not only Markov's inequality but also many other results hinge upon the basic inequalities of Schur and Bernstein. Thus, there is a well-founded interest in improved versions or sharpened inequalities of Schur and Bernstein types for various subclasses of polynomials. An important class of interest is the Bernstein polynomials of fixed sign, that is, the so-called "Lorentz class":

$$
\mathscr{L}:=\left\{p \in \mathscr{P}_{n}: p(x) \neq 0(x \in I)\right\}
$$

Our interest here is the Schur-type inequality for the Lorentz class.

\section{Previous results for the Bernstein-Lorentz class}

For $p \in \mathscr{L}$, that is, for real polynomials $p$ strictly positive (or strictly negative) on the open unit interval $I:=(-1,1)$, a so-called Lorentz representation is possible, see, for example, [3, volume II, page 83, Aufgabe 49]. Actually, Lorentz [4] considered polynomials having the representation

$$
p(x)=\sum_{k=0}^{d} a_{k}(1-x)^{k}(1+x)^{d-k} \quad\left(a_{k} \geq 0(k=1, \ldots, d)\right),
$$

where $d \in \mathbb{N}$ could be any natural number depending on $p \in \mathscr{P}$. Polynomials of this type were used by Lorentz [4] and others in various questions of approximation theory such as approximation by incomplete polynomials, shape preserving approximation, and 
polynomials with integer coefficients. Regarding these, we refer to $[1,2,4-8]$ and the references therein.

The study of the Lorentz class (1.6), the Lorentz representation (2.1), and the "Lorentz degree" $d=d(p)$-defined as the minimal possible degree $d$ of such a representation of the polynomial - is connected to another basic area of interest. Namely, the general idea behind the representation (2.1) is to exhibit the nonnegative polynomial $p \in \mathscr{P}_{n}$ as the positive (nonnegative) linear combination of positive (nonnegative) polynomials $q_{k}^{d}(x):=(1-x)^{k}(1+x)^{d-k}$.

The positive elements $q_{k}^{d}$ form a basis of $\mathscr{P}_{d}$, and (2.1) is a positive representation, that is, a representation with all coefficients $a_{k} \geq 0$. Do all $p \geq 0, p \in \mathscr{P}_{d}$ have the positive representation (2.1)? It is easy to see that the answer to this question is negative. However, such questions lead to other interesting problems, and the whole issue is a vast field of investigations embedded into the theory of Banach lattices and positive bases, see, for example, [9-11]. In particular, these general results show that $\mathscr{P}_{n}$ does not have a positive basis at all, and, moreover, any subspace of $\mathscr{P}_{n}$ with a positive basis has dimension at most $\lfloor n / 2\rfloor$. For these questions, we refer the reader to [12].

Another related matter is the theory of positive operators, in particular, Bernstein operators:

$$
B_{n}(f, x):=\sum_{k=0}^{n} f\left(\frac{2 k-n}{n}\right)\left(\begin{array}{l}
n \\
k
\end{array}\right)(1-x)^{k}(1+x)^{n-k}
$$

Clearly, $B_{n}$ maps $C(I)$ to $\mathscr{P}_{n}$, and for $f \geq 0, B_{n}(f) \geq 0$, that is, $B_{n}(f) \in \mathscr{L}^{+}$, where $\mathscr{L}^{+}:=\left\{p \in \mathscr{P}:\left.p\right|_{I}>0\right\}$. The Bernstein operators are used extensively in the theory of approximation, in particular, for their shape-preserving properties.

Now if $p \in \mathscr{P}_{n}, p \geq 0$ were a fixed-point of $B_{n}$, by comparing (2.1) and (2.2), we could deduce $p \in \mathscr{L}^{+}$and $d(p) \leq n$. Since not all $p \in \mathscr{P}_{n} \cap \mathscr{L}^{+}$have Lorentz degree $d(p) \leq n$, we see that $\left.B_{n}\right|_{\mathscr{P}_{n} \cap \mathscr{L}^{+}}$cannot be identity. In other words, it turns out that the Bernstein operator is not a projection on the set $\mathscr{P}_{n}$. This in turn explains the shortcomings with respect to order of approximation compared to projective operators (like, e.g., the de la Vallée Poussin operator).

Erdélyi and Szabados proved Schur- and Bernstein-type inequalities for these polynomials using their Lorentz degree instead of the ordinary algebraic degree. That brings into focus the question of determining, or at least estimating, the Lorentz degree.

However, estimating the Lorentz degree of a polynomial $p \in \mathscr{L}$ is usually a complicated matter. There are estimates of $d(p)$ in terms of the zero-free region of $p$ described in $[5,13]$. Here we restrict our attention to the most appealing result of this type, attributed to Lorentz, see $[13,14]$.

Theorem 2.1 (Lorentz). Let $p \in \mathscr{L}$. If $\left.p\right|_{D} \neq 0$, then one has $d(p)=\operatorname{deg}(p)$, the ordinary degree.

The reason to pursue estimates of the Lorentz degree is that there are variants of Schur's (and also Bernstein's and Markov's) inequalities to Lorentz polynomials with the Lorentz degree taking over the role of the ordinary algebraic degree. Erdélyi and Szabados [13] (see also [1, E.14, page 436]) have proved the following theorem. 
Theorem 2.2 (Erdélyi-Szabados). Let $p \in \mathscr{L}$ have Lorentz degree $d=d(p)$. Then for any $\alpha>0$ one has

$$
\|p\| \leq \frac{(d+2 \alpha)^{d+2 \alpha}}{(4 \alpha)^{\alpha}(d+\alpha)^{d+\alpha}}\left\|p(x)\left(1-x^{2}\right)^{\alpha}\right\| \quad(p \in \mathscr{P} \cap \mathscr{L}, d=d(p)) .
$$

Observe that here the "Schur constant" is of the order $d^{\alpha}$, and in case $\alpha=1 / 2$, it becomes $\sqrt{d}$, which is a considerable improvement compared to (1.2) provided $d$ is not much larger than $n$. In particular, combining Theorems A and B gave to Erdélyi and Szabados [13] the following theorem.

Theorem 2.3 (Erdélyi-Szabados). Let $p \in \mathscr{L} \cap \mathscr{P}_{n}$ and assume that $\left.p\right|_{D} \neq 0$. Then for any $\alpha>0$ one has

$$
\|p\| \leq \frac{(n+2 \alpha)^{n+2 \alpha}}{(4 \alpha)(n+\alpha)^{n+\alpha}}\left\|p(x)\left(1-x^{2}\right)^{\alpha}\right\| .
$$

Erdélyi and Szabados exhibit the sharpness of (2.4) as well. They also note that their method is bound to use positivity of $p \in \mathscr{L}$ and the result of Theorem A for the Lorentz degree, while formally their end result does not refer to Lorentz degree at all: the formulation of their results on these inequalities does not even need the notion of Lorentz degree and Lorentz representation for this special subclass. Hence they comment: "A direct proof of this statement would be interesting."

\section{Results}

Here we will show that it is possible to obtain Theorem C directly, using only nonvanishing of $p$ on $d$. Moreover, we investigate the similar questions for complex polynomials, where the above convex representation is not available. It turns out that the Schur-type inequalities extend to the complex case unchanged for all $p \in \mathscr{P}_{n}^{c}$ (and thus without assuming any positivity property at all), with the only assumption of nonvanishing in $D$. This is somewhat unexpected, as an example of Halász already established that as regards Bernstein- and Markov-type inequalities, only worse estimates can be obtained for complex polynomials [15], [1, page 447].

We formulate the following.

Theorem 3.1. Let $\phi(t):[0,1] \rightarrow(0, \infty)$ be any decreasing, continuous weight function. Consider a polynomial $p \in \mathscr{P}_{n}^{c}$ and suppose that $\left.p\right|_{D} \neq 0$. Then

$$
\|p(x)\| \leq \frac{2^{n}}{(1+a)^{n} \phi(a)}\|p(x) \phi(|x|)\|=\frac{2^{n}}{\left\|(1+|x|)^{n} \phi(|x|)\right\|}\|p(x) \phi(|x|)\|
$$

with $a \in[0,1]$ being any point of maximum of $\phi(t)(1+t)^{n}$ on $[0,1]$. Moreover, equality occurs only for the polynomials $p(x)=c(1 \pm x)^{n}$ with $c \in \mathbb{C}$ arbitrary.

Corollary 3.2. Let $p \in \mathscr{P}_{n}^{c}$ and suppose that $\left.p\right|_{D} \neq 0$. Then (2.4) holds true for any parameter $\alpha>0$. Moreover, equality occurs only for the polynomials $p(x)=c(1 \pm x)^{n}$ with $c \in \mathbb{C}$ arbitrary. 


\section{Proof of Theorem 3.1}

LeMma 4.1. For arbitrary $z \notin D$ and $0<a<1$ one has

$$
\left|\frac{z-x}{z-a}\right| \leq \frac{2}{1+a} \quad(\forall x \in[a, 1])
$$

Moreover, equality can occur in (4.1) only if $z=-1$ and $x=1$.

Proof. In case $\mathfrak{R} z \geq(1+a) / 2$, we have $|z-x| \leq \max _{x \in[a, 1]}|z-x|=\max (|z-a|, \mid z-$ $1 \mid)=|z-a|$, because for $\mathfrak{R} z \geq(1+a) / 2$ also $|z-a| \geq|z-1|$ holds. Hence in this case (4.1) follows even with $1<2 /(1+a)$ on the right-hand side.

In case $\Re z<(1+a) / 2$, we have, similarly to the above, $|z-x| \leq|z-1|$. Let us consider now the map $f(z):=(z-1) /(z-a)$. This is a rational linear map of $\hat{\mathbb{C}} \rightarrow \widehat{\mathbb{C}}$ assuming real values on $\mathbb{R}$, hence it is also symmetric to the real axis. Moreover, $f$ maps the set of all circles and lines to itself, $f(\infty)=1, f(1)=0, f(a)=\infty$, and $f(-1)=2 /(1+a)$. It follows that the image of the unit circle $C=\partial D$ will be the circle $K$ symmetric to $\mathbb{R}$ and going through the points 0 and $2 /(1+a)$, that is, the circle with center $1 /(1+a)$ and radius $r:=1 /(1+a)$. Moreover, the domain outside of $D$ is mapped onto the interior disk $B$ of $K=\partial B$, since $f(\infty)=1 \in(0,2 /(1+a)) \subset B$. However, $B \subset D(0,2 /(1+a))$, the disk centered at the origin and of radius $2 /(1+a)$. Thus, for all $z \notin D$ the image satisfies $f(z) \in B$ and therefore $|f(z)| \leq 2 /(1+a)$. Consequently, we conclude in this case again that

$$
\left|\frac{z-x}{z-a}\right| \leq\left|\frac{z-1}{z-a}\right|=|f(z)| \leq \frac{2}{1+a}
$$

Moreover, in case $\mathfrak{R} z \geq(1+a) / 2$, there holds a strict inequality, and in case $\mathfrak{R} z<(1+$ a) $/ 2,|z-x|=|z-1|$ entails $x=1$, and $|f(z)|=2 /(1+a)$ entails $z=-1$. Thus, the assertion regarding case of equality follows, too.

Remark 4.2. The above proof follows mapping properties and deduces the estimate from geometrical facts. We thank the referee for pointing out an even quicker, purely algebraic proof, which, however, does not reveal how one may find the right assertion. That explains our choice of keeping the first argument, while the reader may recover himself the latter.

Proof of Theorem 1. Take any parameter $0<a<1$, and consider the polynomial

$$
P_{n}(x):=(1+x)^{n}
$$

Plainly, for any $p(x)=\prod_{j=1}^{n}\left(x-z_{j}\right)$, where for all $j=1, \ldots, n$ we have $\left|z_{j}\right| \geq 1$, we have

$$
\begin{aligned}
& \sup _{x \in[a, 1]}|p(x)| \\
& \quad=\sup _{a \leq x \leq 1}\left|\prod_{j=1}^{n}\left(x-z_{j}\right)\right|=\sup _{a \leq x \leq 1} \prod_{j=1}^{n}\left|\frac{x-z_{j}}{a-z_{j}}\right| \cdot|p(a)| \leq\left(\frac{2}{1+a}\right)^{n}|p(a)|=\frac{P_{n}(1)}{P_{n}(a)}|p(a)| ;
\end{aligned}
$$


hence

$$
\sup _{x \in[a, 1]}|p(x)| \leq \frac{P_{n}(1)}{P_{n}(a) \phi(a)}|p(a) \phi(a)| \leq \frac{P_{n}(1)}{P_{n}(a) \phi(a)}\|p(x) \phi(x)\|_{[0,1]} .
$$

On the other hand, for $0 \leq x \leq a$ we trivially have

$$
|p(x)| \leq \frac{1}{\phi(a)}|\phi(x) p(x)| \leq \frac{1}{\phi(a)}\|p(x) \phi(x)\|_{[0,1]} .
$$

Combining (4.5) and (4.6) we obtain

$$
\sup _{x \in[0,1]}|p(x)| \leq \frac{P_{n}(1)}{P_{n}(a) \phi(a)}\|p(x) \phi(x)\|_{[0,1]},
$$

and applying this also to $p(-x)$ we finally get

$$
\|p\|_{[-1,1]} \leq \frac{P_{n}(1)}{P_{n}(a) \phi(a)}\|p(x) \phi(|x|)\|_{[-1,1]} .
$$

Note that (4.8) actually means also that

$$
\max \left\{\frac{\|p\|_{[-1,1]}}{\|p(x) \phi(|x|)\|_{[-1,1]}}: p \in \mathscr{P}_{n}^{c},\left.p\right|_{D} \neq 0\right\}=\frac{\left\|P_{n}\right\|}{\left\|P_{n}(x) \phi(x)\right\|_{[0,1]}},
$$

because (4.8) holds for all $0<a<1$ and hence the maximum can be taken all over $0<a<1$.

Suppose now that we have equality in the statement of the theorem, that is, in (3.1). Since (4.8) was a consequence of (4.7) and its application to $p(-x)$, case of equality occurs only if (4.7) holds with equality either for $p(x)$ or for $p(-x)$. Suppose, for example, that we have equality in (4.7) for $p(x)$, which implies equality also in (4.4) and (4.5) as well. Equality in (4.4) in turn yields $\left|p\left(x_{0}\right)\right|=\left(P_{n}(1) / P_{n}(a)\right)|p(a)|=(2 /(1+a))^{n}|p(a)|$ for the maximum point $x_{0} \in[a, 1]$ of $p$, and now the equality part of the assertion of Lemma 4.1 implies $x_{0}=1$ and $z_{j}=-1(j=1, \ldots, n)$ for all roots of $p$. But this proves $p(x)=c(1+x)^{n}$, and in case of equality for $p(-x)$, we similarly obtain $p(x)=c(1-x)^{n}$. This concludes the proof.

Proof of Corollary 2. Computing the norm on the right-hand side of (4.9) for $\phi(x)=$ $\left(1-x^{2}\right)^{\alpha}$ — that is, equivalently, taking $a=n /(n+2 \alpha)$ in (4.8) - yields

$$
\frac{\|p\|}{\left\|p(x)\left(1-x^{2}\right)^{\alpha}\right\|} \leq \frac{2^{n}}{(1+n /(n+2 \alpha))^{n}\left(1-n^{2} /(n+2 \alpha)^{2}\right)^{\alpha}}=\frac{(n+2 \alpha)^{n+2 \alpha}}{(n+\alpha)^{n+\alpha}(4 \alpha)^{\alpha}},
$$

which proves (2.4).

\section{Remarks and examples}

Comparing our proof with that of Erdélyi and Szabados, we can realize that the standard approach is to make use of the convex combination (2.1). Denote the set of positive 
Lorentz polynomials of Lorentz degree not exceeding $d$, or ordinary degree not exceeding $n$ by $\mathscr{L}_{+}^{d}$ and by $\mathscr{L}_{+n}$, respectively. It is obvious that $\mathscr{L}_{+}^{d}, \mathscr{L}_{+n}$, and $\mathscr{L}_{+}$are convex sets. Using convexity of $\mathscr{L}_{+}^{d}$, that is, working out proofs for the basis functions $q_{k, d}(x)$ and then adding the results, is a convenient method for real Lorentz polynomials. However, departing real polynomials, we necessarily need complex coefficients, and for $\mathscr{P}^{c} \cap \mathscr{L}$ similar arguments do not work. It turns out that not even the set

$$
\mathscr{P}_{n}^{c}(D):=\left\{p \in \mathscr{P}_{n}^{c}:\left.p\right|_{I}>0,\left.p\right|_{D} \neq 0\right\}
$$

is convex; hence convex combinations cannot be used directly in this setting.

Example 5.1. Let $0<a<1, w:=a+i \sqrt{1-a^{2}}$, and consider the polynomials

$$
\begin{aligned}
p(x) & :=(1-x)\left(x^{2}-2 a x+1\right)=(1-x)(x-w)(x-\bar{w}) \\
& =-x^{3}+(1+2 a) x^{2}-(2 a+1) x+1 \\
q(x) & :=1+x+x^{2}+x^{3}=(1+x)\left(1+x^{2}\right)=(x+1)(x+i)(x-i) .
\end{aligned}
$$

Then $p, q \in \mathscr{P}_{3}^{c}(D)$, but for $r:=(1 / 2) p+(1 / 2) q$ one has $r \notin \mathscr{P}_{3}^{c}(D)$, hence $\mathscr{P}_{3}^{c}(D)$ is not convex.

Indeed, both $p$ and $q$ have zeros of absolute value 1 only, so they belong to $\mathscr{P}_{3}^{c}(D)$. Moreover, for

$$
r(x)=\frac{p(x)+q(x)}{2}=(1+a) x^{2}-a x+1
$$

we obviously have $r \in \mathscr{L}_{+}\left(\mathscr{L}_{+}\right.$is convex $)$. On the other hand, the roots of $r(x)$ are

$$
x_{1,2}=\frac{a \pm \sqrt{a^{2}-4(1+a)}}{2(1+a)}=\frac{a \pm i \sqrt{4+4 a-a^{2}}}{2(1+a)} .
$$

Observe that $4+4 a-a^{2}>4>0$ for all $a \in(0,1)$. Now we can compute

$$
\left|x_{1,2}\right|^{2}=\frac{a^{2}+4+4 a-a^{2}}{4(1+a)^{2}}=\frac{1}{1+a} \leq 1,
$$

hence $\left|x_{1,2}\right|=1 / \sqrt{1+a}<1, x_{1,2} \in D$, and $r \notin \mathscr{P}_{n}^{c}(D)$ for any $n \in \mathbb{N}$.

Note that in this example both $p$ and $q$ have degree 3 , and by Theorem A and $p, q \in$ $\mathscr{P}_{3}^{c}(D)$ their Lorentz degree is 3 . Consequently, by convexity of $\mathscr{L}_{+}$and $\mathscr{L}_{+}^{d}$, we must have $d(r) \leq 3$, while $d(r) \geq \operatorname{deg} r=2$. To decide the exact value of $d(r)$, note that $(1+x)^{2}$, $1-x^{2}$, and $(1-x)^{2}$ form a basis of $\mathscr{P}_{2}$, and easy linear algebra gives $r(x)=(1 / 2)(1+x)^{2}+$ $((1+a) / 2)(1-x)^{2}-(a / 2)\left(1-x^{2}\right)$, whence the unique degree 2 representation is not positive and the Lorentz degree cannot be 2 . Actually, $d(r) \neq \operatorname{deg} r$ already follows from [13, Theorem 2(ii)] or [13, Proposition, page 117]. Whence $d(r)=3$, and the corresponding representation is easily obtained from those of $p$ and $q$. 
The following comment was offered by Tamás Erdélyi.

Remark 5.2 (Erdélyi). As regards Schur's inequality, we have a better than general bound (2.4) at least for the class $\mathscr{P}_{n}^{c}(D)$. In fact, this can also be obtained from the real case, that is, from Theorems B and A, independently of Theorem 3.1 or Corollary 3.2.

Indeed, let $p \in \mathscr{P}_{n}^{c}$ such that $p(z) \neq 0$ for $z \in D$. Consider also

$$
\tilde{p}(z):=\overline{p(\bar{z})}=\prod_{j=1}^{n}\left(z-\overline{z_{j}}\right)\left(p(z):=\prod_{j=1}^{n}\left(z-z_{j}\right)\right)
$$

and take $p^{*}(z):=p(z) \tilde{p}(z)$. Obviously, $p^{*} \in \mathscr{P}_{2 n}$ and $p^{*} \in \mathscr{L}_{+2 n}$, too. Applying Theorem B with power $\alpha^{*}:=2 \alpha$ to $p^{*}$ of degree $n^{*}:=2 n$, we get

$$
\begin{aligned}
\|p\|^{2} & =\left\|p^{*}\right\|=\|p \tilde{p}\| \leq \frac{(2 n+4 \alpha)^{2 n+4 \alpha}}{(8 \alpha)^{2 \alpha}(2 n+2 \alpha)^{(2 n+2 \alpha)}}\left\|p(x) \tilde{p}(x)\left(1-x^{2}\right)^{2 \alpha}\right\| \\
& =\left(\frac{(n+2 \alpha)^{n+2 \alpha}}{(4 \alpha)^{\alpha}(n+\alpha)^{n+\alpha}}\left\|p(x)\left(1-x^{2}\right)^{\alpha}\right\|\right)^{2} .
\end{aligned}
$$

However, for the Bernstein and Markov inequalities in the generality of complex polynomials not vanishing in $D$, we have substantially worse factors, see [1, page 474] and [15]. The example of Halász below shows what we can expect at most.

Example 5.3 (Halász). Let $m$ be chosen as $[(n-1) / 2]$, so that $2 m+1 \leq n \leq 2 m+2$. Define the $\operatorname{deg} P=n$ polynomial $P$ as

$$
P(z):=(z-1) \prod_{j=1}^{m}\left(z-e^{2 \pi i j /(2 m+1)}\right)^{2} .
$$

Then $\left.P\right|_{D} \neq 0,\|P\|_{D}=2=|p(-1)|$, and $p^{\prime}(-1) \gg c n \log n$. Moreover, for any $x \in[-1,1]$, we also have $p^{\prime}(x)>c n \log \left(e /\left(1-x^{2}\right)\right)$ whenever this is smaller than $c n \log n$.

Consequently, no better bound, than $c \min \left(n \log n ; n \log \left(e /\left(1-x^{2}\right)\right)\right.$, is valid in the Markov and Bernstein inequalities, even if we restrict to $\mathscr{P}_{n}^{c}(D)$.

The (essentially standard) calculation showing these lower estimates can be found, for example, in [15] or [1, page 447]. These are indeed the right factors as the corresponding upper estimation is proved, for example, in [15].

A standard way of proving Markov-type inequalities is to combine Bernstein inequalities with Schur inequalities. Of course, to get a sharp Markov estimate we must combine sharp Bernstein and sharp Schur inequalities as well. Thanks to the general form (with any monotone $\phi(x)$ ) of our formulation of the Schur-type inequality Theorem 3.1, here we can indeed deduce the Markov bound from the corresponding Bernstein inequality. Indeed, the known Bernstein-type estimate (see [15, Theorem 2.1]) says that

$$
\left|p^{\prime}(x)\right| \leq n \log \frac{e}{1-x^{2}} \quad\left(|x|<1, p \in \mathscr{P}_{n}^{c}(D)\right)
$$


and applying the Schur inequality (3.1) to $p^{\prime}(x)$ and $\phi(x):=\log ^{-1}\left(e /\left(1-x^{2}\right)\right)$ we obtain

$$
\left\|p^{\prime}(x)\right\| \leq \frac{2^{n}}{\left\|(1+x)^{n} \phi(x)\right\|}\left\|\phi(x) p^{\prime}(x)\right\| \leq \frac{2^{n}}{\left|\left(1+x_{0}\right)^{n} \phi\left(x_{0}\right)\right|}\left\|\phi(x) p^{\prime}(x)\right\|
$$

with arbitrary $x_{0} \in I$. Choosing $x_{0}:=1-2 / n$, say, we thus obtain $\left\|p^{\prime}\right\| \leq C \log n\left\|\phi p^{\prime}\right\|$ and this can be estimated by the above Bernstein inequality (5.9) as $\leq C n \log n$.

Note that given the logarithmic weight in the complex case, restricting to weights $\left(1-x^{2}\right)^{\alpha}$ would bring by itself the loss of the possibility of this deduction.

\section{Acknowledgments}

The author was supported in part by the Hungarian National Foundation for Scientific Research, Project no. T-049301 and K-61908. This work was accomplished during the author's stay in Paris under his Marie Curie fellowship, contract no. MEIF-CT-2005-022927.

\section{References}

[1] P. Borwein and T. Erdélyi, Polynomials and Polynomial Inequalities, vol. 161 of Graduate Texts in Mathematics, Springer, New York, NY, USA, 1995.

[2] G. V. Milovanović, D. S. Mitrinović, and Th. M. Rassias, Topics in Polynomials: Extremal Problems, Inequalities, Zeros, World Scientific, River Edge, NJ, USA, 1994.

[3] Gy. Pólya and G. Szegö, Aufgaben und Lehrsätze aus der Analysis, Vol. II, vol. 20 of Die Grundlehren der matehmatischen Wissenschaften in Einzeldarstellungen, Springer, Berlin, Germany, 1925.

[4] G. G. Lorentz, "The degree of approximation by polynomials with positive coefficients," Mathematische Annalen, vol. 151, no. 3, pp. 239-251, 1963.

[5] T. Erdélyi, "Estimates for the Lorentz degree of polynomials," Journal of Approximation Theory, vol. 67, no. 2, pp. 187-198, 1991.

[6] K. A. Kopotun, "Uniform estimates of monotone and convex approximation of smooth functions," Journal of Approximation Theory, vol. 80, no. 1, pp. 76-107, 1995.

[7] K. A. Kopotun, D. Leviatan, and I. A. Shevchuk, "Convex polynomial approximation in the uniform norm: conclusion," Canadian Journal of Mathematics, vol. 57, no. 6, pp. 1224-1248, 2005.

[8] K. Kopotun, D. Leviatan, and I. A. Shevchuk, "Coconvex approximation in the uniform norm: the final frontier," Acta Mathematica Hungarica, vol. 110, no. 1-2, pp. 117-151, 2006.

[9] I. A. Polyrakis, "Finite-dimensional lattice-subspaces of $C(\Omega)$ and curves of $\mathbf{R}^{n}$," Transactions of the American Mathematical Society, vol. 348, no. 7, pp. 2793-2810, 1996.

[10] I. A. Polyrakis, "Lattice-subspaces of $C[0,1]$ and positive bases," Journal of Mathematical Analysis and Applications, vol. 184, no. 1, pp. 1-18, 1994.

[11] I. A. Polyrakis, "Minimal lattice-subspaces," Transactions of the American Mathematical Society, vol. 351, no. 10, pp. 4183-4203, 1999.

[12] B. Farkas and S. Gy. Révész, "Positive bases in spaces of Polynomials," preprint, 2007 http://arxiv.org/abs/0708.2883.

[13] T. Erdélyi and J. Szabados, "On polynomials with positive coefficients," Journal of Approximation Theory, vol. 54, no. 1, pp. 107-122, 1988. 


\section{Journal of Inequalities and Applications}

[14] J. T. Scheick, "Inequalities for derivatives of polynomials of special type," Journal of Approximation Theory, vol. 6, no. 4, pp. 354-358, 1972.

[15] T. Erdélyi, "Markov-Bernstein type inequalities for constrained polynomials with real versus complex coefficients,” Journal d’Analyse Mathématique, vol. 74, pp. 165-181, 1998.

Szilárd Gy. Révész: Alfréd Rényi Institute of Mathematics, Hungarian Academy of Sciences, P.O. Box 127, 1364 Budapest, Hungary

Email address: revesz@renyi.hu 